\title{
Prediction and evaluation of everyday memory in neurological patients
}

\author{
AMY HERSTEIN GERVASIO \\ Hamilton College, Clinton, New York \\ and \\ MATTHEW J. BLUSEWICZ \\ Veterans Administration Medical Center, Martinez, California \\ and University of California, Davis, California \\ (Douglas J. Herrmann, Sponsor)
}

\begin{abstract}
Current research suggests that normal adults have only moderate insight or awareness of their memory functioning, although their insight is better than that of neurological patients with memory loss. Using the Self-Assessment of Laboratory Tasks (SALT), Herrmann, Grubs, Sigmundi, and Grueneich (1986) found that when normal adults had experience with specific memory tasks, they evaluated memory performance much better than they predicted it. The present research investigated the ability of neurological patients and hospitalized controls to predict and evaluate everyday memory performance using the SALT. Contrary to conventional wisdom, the neurological patients with memory loss showed awareness of loss; they reported their performance to be at a lower level than did other subjects. Also, the memory-loss patients increased their evaluation scores as much as normals; the agreement between self-assessment and actual performance was greater after performance.
\end{abstract}

Although memory loss and metamemory are of interest to both clinical and cognitive psychologists, the methods used for studying memory in the two fields have only recently begun to overlap (Baddeley, 1981). The general memory tests conventionally used in clinical settings, such as the Wechsler Memory Scale (Wechsler, 1945) and the Memory-for-Designs Test (Graham \& Kendall, 1960), do not address specific memory complaints of patients and are plagued with psychometric inadequacies, including lack of discriminant validity (Lezak, 1983). The applicability of traditional laboratory tests of memory for clinical populations and their relation to everyday tasks have been questioned (Sunderland, Harris, \& Baddeley, 1983; Zelinski, Gilewski, \& Thompson, 1980). Possible adjuncts to traditional assessment procedures may be found in the study of "everday" memory and metamemory.

Beliefs about memory have usually been assessed through self-report questionnaires, a method with high reliability and often relatively low validity (Herrmann, 1982; Morris, 1983). Using one such measure of everyday memory, called the Short Inventory of Memory Experiences (SIME), Herrmann and Neisser (1978) found that most people report memory problems to some degree. Several studies have reported that neurological patients are not able to assess their own memories using such tests

We thank Douglas Herrmann for his comments on an earlier draft of this manuscript. Requests for reprints should be sent to Amy Herstein Gervasio, Department of Psychology, Hamilton College, Clinton, NY 13323. as the SIME (Sunderland, Harris, \& Baddeley, 1983) and that the spouses of head-injured patients assess the patients' memories more accurately than the patients themselves (Baddeley, Sunderland, \& Harris, 1982; Sunderland, Harris, \& Gleave, 1984). However, with temporal lobectomy patients, Bennett-Levy, Polkey, and Powell (1980) found that those clinical factors that correlate with memory performance also predict self-report scores on everyday memory surveys.

The ability to accurately assess one's memory may differ with age. Zelinski et al. (1980) found that everyday memory questionnaires correlated with performance of laboratory tasks in older people, but not in young adults. They argued that older adults may be more sensitized to memory failures than college students, and that questionnaires may predict performance only when it is below peak level.

Herrmann, Grubs, Sigmundi and Grueneich (1986) argued that the more specific the memory questionnaire is, the better the predictive and postdictive validity. They presented college students with the Self-Assessment of Laboratory Tasks (SALT), in which students predicted their memory performance on everyday memory tasks. Although correlations between predictions and performance on those tasks were low, the correlations between performance and evaluations of the performance (as measured by a second administration of the SALT) were higher. Although students were unable to assess their memories accurately, they could use the feedback gained 
from doing the tasks to reassess their memory performance.

Extending the work on prediction and evaluation to clinical populations seems useful for three reasons: (1) tests of everyday memory may correspond to memory complaints that patients present, (2) it may be important to know how accurate memory complaints are, and (3) the ability of a patient to evaluate memory performance may be useful when designing a cognitive rehabilitation program. Accordingly, this research explores (1) the relationship between traditional tests of memory used in clinical settings, such as the Wechsler Memory Scale (WMS), and surveys and performances of everyday memory tasks, and (2) the ability of neurological patients and controls to predict and evaluate their own memory performance. This is a partial replication of the work on memory awareness in college students conducted by Herrmann et al. (1986).

Hypotheses were (1) that although controls would perform better than patients, both neurological patients whose major complaint was memory loss and hospitalized controls would have difficulty predicting memory performance; (2) that both groups would be able to more accurately evaluate than predict their performance; and (3) that evaluation in control patients would be more accurate.

\section{METHOD}

\section{Subjects}

The subjects were 21 male and 1 female neurological patients at a Veterans Administration hospital. Eleven of these patients were inpatients on a neurological ward at the time of testing, and 11 were outpatients receiving similar rehabilitation therapy on the same ward. The patients were suggested as being suitable for the study by neurologists and other rehabilitation staff. All neurological patients had brain damage as documented by electroencephalogram, CAT scan, PET scan, NMR, injury, and so forth. Seven patients had suffered from stroke, 7 from head trauma, 3 from early stages of Alzheimer's disease (diagnosed within the last 6 months), 2 from multiple sclerosis with mental status changes, and 3 from tumors or other brain damage.

Neurological patients were divided into two groups: 15 patients with a history of memory loss and 7 patients without a history of memory loss. Each patient's history was determined through chart records of neurological exams and reports of staff working with the patient. The average age of the memory-loss group was $49.13(S D=15.08)$, and the average age of the no-loss group was $47.2(S D=17.14)$.

Control subjects were 16 males hospitalized for orthopedic problems, with an average age of $47(S D=15.83)$. The age range of all three groups was $23-69$, and patients were roughly matched for age and education among the control and neurological groups. All subjects signed consent forms; the research was approved by a human subjects committee.

\section{Procedure}

The subjects were asked to predict their performance on the SALT (Herrmann et al., 1986), which asks subjects to predict their memory performance on nine specific tasks. Five of the items correspond to items on the WMS: memory passages, forward and backward digit span, visual reproduction, and paired-associate learning. The remaining items are memory for names and faces, placing cities on a state map, placing items in a room plan, and tracing directions on a map from oral instructions. (For the purposes of clarity, the actual prediction scores on the SALT will be referred to as P-SALT scores.)
After taking the SALT, the subjects then took the WMS (Form II) and the performance tests corresponding to the remaining SALT items, as well as the Memory for Designs Test (MFD; Graham \& Kendall, 1960). After the battery, they evaluated their performance using a pasttense version of the SALT, termed the E-SALT. Finally, after the E-SALT, subjects were given the SIME (Herrmann \& Neisser, 1978). Thus, each subject produced nine predictive (P-SALT) scores, a total of seven full-test performance scores and five subtest scores from the WMS, and nine evaluative (E-SALT) scores. (The average administration time for each subject was $2 \frac{1 / 2}{2}$.)

\section{RESULTS}

\section{Descriptive Data}

The WMS and MFD, which require experience and inference in scoring, were scored by the second author, who was blind to the condition of the subjects and blind to testing.

Both the control and the no-loss groups scored higher than average on the WMS $(M=114.57, S D=15.39$, for the no-loss group; $M=109.37, S D=14.99$, for the control group), and the memory-loss group scored an average of $86.6(S D=15.34)$, which is $1 S D$ lower than the norm. The average WMS score across all subjects was $101.36(S D=19.14)$, indicating that the sample was similar to national norms. Similarly, the memory-loss group showed deficient performance on the MFD (Taylor, 1961).

\section{Statistical Analysis}

The SIME and the WMS were significantly and moderately correlated across all subjects $(r=.470, p \leq .002)$, as were the SIME and MFD $(r=.478, p \leq .002)$.

Differences in test performance. A multivariate analysis of variance (MANOVA) revealed significant differences for group performance on the SIME, WMS, and MFD, using Wilk's criterion $[F(6,66)=5.00, p \leq$ $.0003]$. The control and the no-loss groups performed significantly better than the memory-loss group (see Table 1). A MANOVA using the nine performance tests corresponding to the SALT items as dependent variables was significant for group, using Wilk's criterion $[F(18,48)$ $=2.19, p \leq .01]$. Duncan's post hoc tests revealed that the memory-loss group performed significantly lower than the no-loss group, which was equal to the control group on four tasks (i.e., names and faces, backward digit span, visual reproduction, and paired-associate learning). The

Table 1

Means and Standard Deviations for Performance Items by Group

\begin{tabular}{|c|c|c|c|c|c|c|}
\hline \multirow[b]{3}{*}{ Item } & \multicolumn{6}{|c|}{ Group } \\
\hline & \multicolumn{2}{|c|}{$\begin{array}{c}\text { No-Loss } \\
(N=7)\end{array}$} & \multicolumn{2}{|c|}{$\begin{array}{c}\text { Memory-Loss } \\
(N=15)\end{array}$} & \multicolumn{2}{|c|}{$\begin{array}{l}\text { Control } \\
(N=16)\end{array}$} \\
\hline & $M$ & $S D$ & $M$ & $S D$ & $M$ & $S D$ \\
\hline SIME & 124.85 & 15.27 & 102.53 & 23.67 & 125.25 & 13.23 \\
\hline WMS & 114.57 & 15.39 & 86.67 & 15.31 & 109.38 & 15.00 \\
\hline MFD & 8.00 & 7.90 & 18.27 & 7.39 & 7.50 & 6.08 \\
\hline
\end{tabular}

Note-Differences between groups are significant at the $p \leq .01$ level. SIME $=$ Short Inventory of Memory Experiences. WMS $=$ Wechsler Memory Scale. MFD = Memory for Designs Test. 
Table 2

Average Correlations of Predictive and Evaluative Items with Performance

\begin{tabular}{|c|c|c|c|c|c|c|}
\hline \multirow{2}{*}{$\begin{array}{c}\text { Group } \\
\text { Variable }\end{array}$} & \multicolumn{2}{|c|}{ No-Loss } & \multicolumn{2}{|c|}{ Memory-Loss } & \multicolumn{2}{|c|}{ Control } \\
\hline & P-SALT/P & E-SALT/P & P-SALT/P & $\overline{\text { E-SALT/P }}$ & P-SALT/P & E-SALT/P \\
\hline Passages & .292 & $.829 \dagger$ & -.029 & .437 & $.641 \dagger$ & $.658 \dagger$ \\
\hline Digits forward & .706 & .611 & -.060 & .346 & $.490 *$ & .348 \\
\hline Digits backward & .285 & $.757 *$ & -.210 & .154 & -.363 & .298 \\
\hline Visual reproduction & .214 & .608 & .217 & $.739 *$ & $.495^{*}$ & $.693 \dagger$ \\
\hline Paired-associates & .307 & .732 & .039 & .304 & $.680 \dagger$ & $.741 \dagger$ \\
\hline Room plan & .375 & .707 & .216 & $.637 \dagger$ & -.191 & $.845 \dagger$ \\
\hline State map & -.070 & .709 & $.751 \dagger$ & $.704+$ & .116 & $.915^{*}$ \\
\hline Oral directions & -.468 & .286 & -.131 & .221 & -.189 & .386 \\
\hline Names and faces & .439 & $.992 \dagger$ & .170 & .298 & .189 & .417 \\
\hline $\begin{array}{l}\text { Average correlations } \\
\text { Overall average }\end{array}$ & .472 & .769 & .181 & .446 & .354 & .641 \\
\hline (all subjects) & .272 & $.598 \dagger$ & & & & \\
\hline
\end{tabular}

no-loss group was significantly superior to the control group (which was, in turn, superior to the memory loss group) on the room plan and forward digit span tests. (More complete tables are available from the author.)

Prediction and evaluation. For the total subject sample and for each of the three groups of patients, correlations were calculated for each P-SALT and performance item and also for each E-SALT and performance item. Average correlations for each group were then calculated (McNemar, 1955) to preclude an inflated number of significant correlations. As can be seen in Table 2, the highest average correlations were for the no-loss-group, closely followed by the control group and by the memoryloss group. There were 16 significant correlations, out of 54, which is almost five times the number expected by chance. For all subjects, there was a significant difference at the $p \leq .01$ level between the average P-SALT/performance correlations $(r=.272)$ and the ESALT/performance correlations $(r=.598)$, indicating that the subjects were better able to evaluate their performance than predict it.

Two difference scores were calculated for each of the nine performance items: the absolute difference between prediction (P-SALT) scores and performance, and the absolute difference between evaluation (E-SALT) scores and performance. The MANOVA for differences between prediction and performance among groups was not significant $[F(18,48)=1.16, p \leq .32]$, using Wilk's criterion. The difference in the evaluation of performance was also not significant $[F(18,48)=1.29, p \leq .23]$.

\section{DISCUSSION}

In contrast to other studies (Sunderland et al., 1983), the present study found significant and moderate correlations ( $r$ of .47 ) between the SIME and performance on the MFD and WMS, when correlations were performed for all subjects. Previous studies using neurological patients report low correlations ( $r$ s around .2-.3) between self-report questionnaires and test performance. These previous studies compute separate correlations for patients and controls rather than an overall correlation for all subjects. If there were more homogeneity of the variance in the scores of any one subject group, then separate analysis could show low correlations even if the overall correlation was moderate or high.
As predicted in Hypothesis 1, controls did perform better than patients. However, results followed those of Herrmann et al. (1986) for prediction and evaluation of specific memory tasks: both neurological patients and controls were only moderately able to predict their own memory performance.

As predicted in Hypothesis 2, correlations between evaluation scores and performance were significantly higher than correlations between prediction and performance scores. Findings suggest that even neurological patients, who are often characterized by their lack of "insight," could take note of their performance to enhance their evaluation scores, at least temporarily. (Clearly, the design of this study could not differentiate between improved insight concerning memory processes over time and enhanced episodic memory related to taking the test.) Thus, coupling memory performance tasks with systematic prediction and evaluation may provide a useful treatment in itself for increasing insight and for giving feedback in rehabilitation programs.

Hypothesis 3, which concerned differential ability of groups to evaluate their performance, did not seem to be confirmed; group difference scores were not significant when all groups were compared. This failure does not necessarily suggest that groups were equally able to evaluate themselves, however, because the correlations between patient performance and evaluation scores were still lower than those of controls. The gain from prediction to evaluation was similar.

Predictive correlations for all groups in this study was generally higher than the $r s$ of .10 found for college students in Herrmann et al.'s (1986) study. As with the older subjects in Zelinski et al.'s (1980) study, the patients in the present study may have been more sensitive to memory loss because they were older, and because patients undergoing rehabilitation may be constantly exhorted to remember things while on the ward.

The trend showing that a small sample of neurological patients without a history of memory loss may be better than controls at predicting and evaluating memory bears further study. Perhaps the "conscious awareness" these patients report feeling makes them more keen observers of their own memories and more able to use the feedback that performance presents. Future research in memory awareness should include larger samples of patients who have more homogeneous disorders, in order to ascertain whether or not individual items on tests of everyday memory may be useful in understanding the nature of specific memory disorder. Finally, focusing on individual items in tests of everyday memory may be useful for designing cognitive rehabilitation strategies.

\section{REFERENCES}

BAdDEley, A. (1981). The cognitive psychology of everyday life. Bulletin of the British Psychological Society, 34, 166-168.

Baddeley, A., Sunderland, A., \& Harris, J. (1982). How well do laboratory based psychological tests predict patients' performance outside the laboratory? In S. Corkin, K. L. Davis, J. H. Growdon, E. Usdin, \& R. J. Wurtman (Eds.), Alzheimer's disease: A report of progress in research (pp. 141-148). New York: Raven Press. 
Bennett-Levy, J., Polkey, C. E., \& Powell, G. E. (1980). Selfreport of memory skills after temporal lobectomy: The effect of clinical variables. Cortex, 16, 543-557.

Graham, F. K., \& Kendall, B. S. (1960). Memory-for-Designs Test: Revised general manual. Perceptual and Motor Skills, 11(Suppl. 2-VIII), 147-188.

Herrmann, D. J. (1982). Know thy memory: The use of questionnaires to assess and study memory. Psychological Bulletin, 92, 434-452.

Herrmann, D. J., Grubs, L., Sigmundi, R., \& Grueneich, R. (1986). Awareness of memory ability as a function of memory experience. Human Learning, 5, 91-107.

HerrmanN, D. J., \& Neisser, U. (1978). An inventory of memory experiences. In P. E. Morris \& R. N. Sykes (Eds.), Practical aspects of memory. New York: Academic Press.

LeZAK, M. (1983). Neuropsychological assessment (2nd ed.). New York: Oxford University Press.

McNemar, Q. (1955). Psychological statistics (2nd ed.). New York: Wiley.

MorRIS, P. E. (1983). The validity of subjective reports on memory. In J. E. Harris \& P. E. Morris (Eds.), Everyday memory, actions and absent-mindedness. London: Academic Press.
Sunderland, A., Harris, J. E., \& Baddeley, A. D. (1983). Do laboratory tests predict everyday memory? Journal of Verbal Learning \& Verbal Behavior, 22, 341-357.

Sunderland, A., Harris, J. E., \& Gleave, J. (1984). Memory failures in everyday life following severe head injury. Journal of Clinical Neuropsychology, 6, 127-142.

TAYLOR, F. R. (1961). A revised scoring system for the Graham-Kendall Memory-for-Designs Test. Unpublished masters thesis, University of Utah, Salt Lake City.

WECHSLER, D. (1945). A standardized memory scale for clinical use. Journal of Psychology, 19, 87-95.

Zelinski, E. M., Gilewski, M. J., \& Thompson, L. W. (1980). Do laboratory tests relate to self-assessment of memory ability in the young and old? In L. W. Poon, J. L. Fozard, L. S. Cermak, D. Arenberg, \& L. W. Thompson, New directions in memory and aging: Proceedings of the George A. Talland Memorial Conference (pp. 519-545). Hillsdale, NJ: Erlbaum.

(Manuscript received for publication January 19, 1988.) 